\title{
TRANSCRITICAL FLOW PAST AN OBSTACLE
}

\author{
R. GRIMSHAW ${ }^{1}$
}

\author{
(Received 28 January, 2011; revised 22 March, 2011)
}

\begin{abstract}
It is well known that transcritical flow past an obstacle may generate undular bores propagating away from the obstacle. This flow has been successfully modelled in the framework of the forced Korteweg-de Vries equation, where numerical simulations and asymptotic analyses have shown that the unsteady undular bores are connected by a locally steady solution over the obstacle. In this paper we present an overview of the underlying theory, together with some recent work on the case where the obstacle has a large width.
\end{abstract}

2000 Mathematics subject classification: primary 74J30.

Keywords and phrases: solitary waves, transcritical flow, undular bore.

\section{Introduction}

In general, fluid flow over an obstacle generates wave trains, which can be found both upstream and downstream depending on the system parameters. The most familiar scenario is for free-surface channel flow, but the same essential features arise in many other fluid flows, such as for internal waves. Our special interest is in the critical case, defined as that when the fluid flow supports a linear long wave speed $c=0$ in the reference frame of the obstacle. When the flow is not critical, linear theory may be used to describe the wave field, and the solution can be obtained using Fourier transforms; see, for instance [25, 32]. For water waves and internal waves, typically stationary lee waves are found downstream for subcritical flow when $c<0$, together with transients propagating both upstream and downstream, while only downstreampropagating transients are found in supercritical flow when $c>0$. However, these linear solutions fail near criticality, as then the wave energy cannot propagate away from the obstacle. In this case it is necessary to invoke weak nonlinearity to obtain a suitable theory, and it is now well established that the forced Korteweg-de Vries $(\mathrm{fKdV})$ equation is an appropriate model. In canonical form it is

$$
-A_{t}-\Delta A_{x}+6 A A_{x}+A_{x x x}+F_{x}(x)=0 .
$$

\footnotetext{
${ }^{1}$ Department of Mathematical Sciences, Loughborough University, Loughborough, LE11 3TU, UK; e-mail: R.H.J.Grimshaw @lboro.ac.uk.

(C) Australian Mathematical Society 2011, Serial-fee code 1446-1811/2011 \$16.00
} 
Here $A$ is a measure of the wave amplitude, $\Delta$ measures the linear long wave speed so that $\Delta>0$ and $\Delta<0$ define the linear supercritical and subcritical regimes respectively, and $F(x)$ is a forcing term representing the obstacle.

The fKdV equation (1.1) is based on an asymptotic multi-scale expansion in which $A \sim \epsilon^{2}, \Delta \sim \epsilon^{2}, F \sim \epsilon^{4}, \partial / \partial x \sim \epsilon, \partial / \partial t \sim \epsilon^{3}$. For water waves the fKdV equation has been derived by several authors [1, 5, 24, 27, 33], and for internal waves by Grimshaw and Smyth [18], Melville and Helfrich [28] and Clarke and Grimshaw [4]. In Appendix A we present a brief summary of the derivation of the $\mathrm{fKdV}$ equation for internal waves. Numerical and asymptotic analytical solutions of the fKdV equation (1.1) by these and other authors have demonstrated that for flow over a localized obstacle of positive polarity (that is, $F(x) \geq 0$ ), the transcritical regime consists of upstream and downstream undular bores connected by a locally steady solution over the obstacle. These theoretical predictions have been confirmed in several laboratory experiments and numerical simulations. The first observations of upstream wave trains were made by Thews and Landweber [31] in ship wave experiments. Subsequently, several surface wave experiments (see Huang et al. [23], $\mathrm{Wu}$ and $\mathrm{Wu}$ [34], Ertekin et al. [14, 15] and Lee et al. [24]) and an interfacial wave experiment by Melville and Helfrich [28] confirmed upstream waves in the transcritical regime. Numerical simulations of the full Euler equations for water waves by Zhang and Chwang [35, 36] for transcritical flow over an obstacle, and for interfacial waves by Grue et al. [21], likewise confirmed the generation of upstream wave trains. While the entire focus of this article is on the fKdV equation, it is pertinent to note that, depending on the physical circumstances, transcritical flows can also be modelled by other forced nonlinear wave equations. For instance, the forced extended Korteweg-de Vries (Gardner) equation, in which a cubic nonlinear term is added to (1.1), has been used to model the transcritical generation of interfacial waves by Melville and Helfrich [28], Marchant and Smyth [26] and Grimshaw et al. [16], the forced $\mathrm{Su}-\mathrm{Gardner}$ equation has been used to study the transcritical generation of finite-amplitude water waves by El et al. [13], and the forced Benjamin-Ono equation has been used to study the transcritical generation of atmospheric internal waves by Porter and Smyth [29] in the context of a study of "Morning Glory" waves. In all of these cases, the same basic methodology described here for the fKdV equation (1.1) has been used for the construction of asymptotic solutions.

In Section 2 we review the asymptotic solution of the fKdV equation (1.1) presented initially by Grimshaw and Smyth [18] and Smyth [30] for flow over a localized obstacle, and recently extended by Grimshaw et al. [19, 20] to flow over a step. This asymptotic solution consists of upstream and downstream undular bores connected by a locally steady solution over the obstacle. Hence in Section 3 we investigate in more detail this local steady solution with an emphasis on the effect of the obstacle width. We conclude in Section 4 with a summary and discussion. In Appendix A we present a brief description of the derivation of the $\mathrm{fKdV}$ equation (1.1) for internal waves in a shear flow, and in Appendix B we describe the asymptotic undular bore solutions of the $\mathrm{KdV}$ equation. 


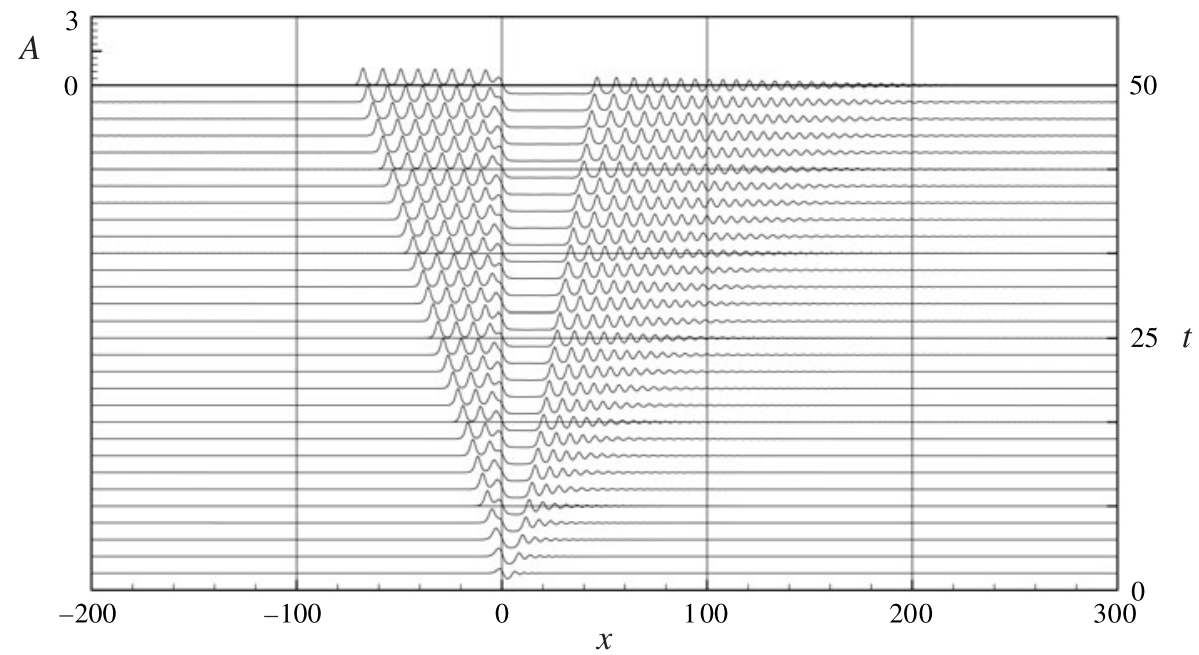

Figure 1. Numerical simulation of the fKdV equation (1.1) for a localized obstacle, with $F_{M}=0.45$ and $\Delta=0.0$.

\section{Unsteady forced solutions}

2.1. Transcritical flow over a localized obstacle First we present a brief summary of the asymptotic theory for a localized obstacle, based on the initial study of Grimshaw and Smyth [18] and Smyth [30]. We suppose that $F(x)$ is positive and localized, that is, $F(x) \geq 0$ for $0<x<L$, with a maximum value of $F_{M}>0$, and is zero otherwise. A numerical simulation is shown in Figure 1 for exact criticality. The solution is characterized by upstream and downstream wave trains connected by a locally steady solution over the obstacle. For subcritical flow $(\Delta<0)$ the upstream wave train weakens, and for sufficiently large $|\Delta|$ detaches from the obstacle, while the downstream wave train intensifies and for sufficiently large $|\Delta|$ forms a stationary lee wave field. On the other hand, for supercritical flow $(\Delta>0)$ the upstream wave train develops into well-separated solitary waves while the downstream wave train weakens and moves further downstream (for more details, see [18-20, 30]).

The origin of the upstream and downstream wave trains can be found in the structure of the locally steady solution over the obstacle. In the transcritical regime this local steady solution $A=A_{s}(x)$ satisfies the equation

$$
-\Delta A_{s}+3 A_{s}^{2}+A_{s x x}+F(x)=C,
$$

which is obtained from (1.1) by setting $A_{t}=0$ and performing one integration, yielding $C$ as an unknown constant of integration. We then seek a solution characterized by a transition from a constant state $A_{-}$upstream $(x<0)$ of the obstacle to a constant state $A_{+}$downstream $(x>L)$ of the obstacle, so that the boundary conditions for (2.1) are

$$
A_{s} \rightarrow A_{ \pm} \text {for } x \rightarrow \pm \infty \text {. }
$$


Assuming that the limiting steady states $A_{ \pm}$have been determined, we see that upstream of the obstacle there is a transition from the zero state as $x \rightarrow-\infty$ to $A_{-}$, while downstream the transition is from $A_{+}$to the zero state as $x \rightarrow \infty$. Each transition is effectively generated in the vicinity of $x=0$, but importantly exists in regions where $F=0$ and hence can be described either by the "undular bore" solutions, or by a rarefraction wave solution of the unforced $\mathrm{KdV}$ equation, as described in Appendix B. A typical undular bore is shown in Figure 10 in Appendix B. For an undular bore to be formed downstream propagating away from the obstacle, $A_{+}<0$; otherwise if $A_{+}>0$, the transition is a rarefraction wave. Upstream, $A_{-}>0$ implies the presence of an undular bore propagating away from the obstacle, while $A_{-}<0$ implies that the transition is a rarefraction wave.

Applying the boundary conditions (2.2),

$$
C=-\Delta A_{ \pm}+3 A_{ \pm}^{2} \quad \text { so that } \Delta=3\left(A_{+}+A_{-}\right),
$$

independently of the details of the obstacle shape $F(x)$. Explicit determination of $A_{+}$ and $A_{-}$requires more knowledge of the obstacle shape $F(x)$ in order to determine $C$, an issue which is taken up in Section 3. Here, however, following the strategy of Grimshaw and Smyth [18] and Smyth [30], we note that in the dispersionless, or "hydraulic", limit when the linear dispersive term in (2.1) is neglected, equation (2.1) reduces to an algebraic equation which determines $A_{s}(x)$ explicitly in terms of $F(x)$. Then $C$ is determined by considering the long-time limit of the forced Hopf equation, obtained from the fKdV equation (1.1) by omitting the linear dispersive term, with the initial condition that $A(x, 0)=0$. The outcome is the criticality condition that $A_{s x} \neq 0$ when $F_{x}=0, F=F_{M}$, and thus $C=F_{M}-\Delta^{2} / 12$. It is then readily shown that

$$
6 A_{ \pm}=\Delta \mp\left(12 F_{M}\right)^{1 / 2} \text {. }
$$

This expression also serves to define the transcritical regime, which is

$$
|\Delta|<\left(12 F_{M}\right)^{1 / 2} \text {. }
$$

As expected, $A_{+}<0, A_{-}>0$, and so the transitions both downstream and upstream are described by undular bores.

The undular bore in $x<0$ is described by (B.3)-(B.6) with $A_{0}=A_{-}$. Hence it occupies the zone

$$
\Delta-4 A_{-}<\frac{x}{t}<\max \left\{0, \Delta+6 A_{-}\right\} .
$$

Note that this upstream wave train is constrained to lie in $x<0$, and hence is only fully realized if $\Delta<-6 A_{-}$. Combining this criterion with (2.4) and (2.5) defines the regime

$$
-\left(12 F_{M}\right)^{1 / 2}<\Delta<-\frac{1}{2}\left(12 F_{M}\right)^{1 / 2},
$$

where a fully developed undular bore solution can develop upstream. On the other hand, the regime $\Delta>-6 A_{-}$, or

$$
-\frac{1}{2}\left(12 F_{M}\right)^{1 / 2}<\Delta<\left(12 F_{M}\right)^{1 / 2}
$$


is where the upstream undular bore is only partially formed and is attached to the obstacle. In this case the modulus $m$ of the Jacobi elliptic function varies from 1 at the leading edge (thus describing solitary waves) to a value $m_{-}<1$ at the obstacle, where $m_{-}$can be found by putting $x=0$ in (B.5).

The undular bore in $x>L$ can also be described by (B.3)-(B.6) with $A_{0}=-A_{+}$, after using the transformation (B.9). Hence it occupies the zone

$$
\max \left\{0, \Delta-2 A_{+}\right\}<\frac{x-L}{t}<\Delta-12 A_{+} .
$$

Here, this downstream wave train is constrained to lie in $x>L$, and hence is only fully realized if $\Delta>2 A_{+}$. Combining this criterion with (2.4) and (2.5) defines the regime (2.8), and so a fully detached downstream undular bore coincides with the case where the upstream undular bore is attached to the obstacle. On the other hand, in the regime (2.7), when the upstream undular bore is detached from the obstacle, the downstream undular bore is attached to the obstacle, with a modulus $m_{+}<1$ at the obstacle, where $m_{+}$can again be found from the counterpart of (B.5) evaluated at $x=L$.

For the case where the obstacle has negative polarity (that is, $F(x) \leq 0,0<x<L$ ), the upstream and downstream wave trains are qualitatively similar. However, the solution in the vicinity of the obstacle remains transient, and this causes a modulation of the "undular bore" solutions. The explanation for this can be found either in the "hydraulic" limit when it can be shown that there is then no locally steady solution (see [18, 30]), or from the analysis of flow over a step, discussed in [19, 20] and summarized in Section 2.2.

2.2. Transcritical flow over a step From numerical simulations of the full Euler equations for free-surface transcritical flow over wide obstacles, Zhang and Chwang [36] showed that a positive step generates only upstream waves while a negative step generates only downstream waves. Subsequently, Grimshaw et al. $[19,20]$ verified this observation in the framework of the fKdV equation (1.1), and also provided a theoretical explanation in terms of the structure of the locally steady solutions over the step. The solution for a wide obstacle can then be regarded as a combination of the solutions over two widely separated steps. Thus, since a positive obstacle is a combination of a positive step and then a negative step, it follows that the front positive step generates an undular bore propagating upstream away from the obstacle, while the rear negative step generates an undular bore propagating downstream, also away from the obstacle. Hence no waves form over the obstacle itself, and locally the solution there becomes steady. However, a negative obstacle (or hole) is a combination of a negative step and then a positive step, and so it follows that the negative step generates an undular bore propagating downstream over the obstacle, while the rear positive step generates an undular bore propagating upstream, also over the obstacle. These wave trains then meet and interact, producing an unsteady solution over the obstacle. Although each wave train may eventually propagate away from the obstacle, at least partially, numerical simulations indicate that the solution remains 


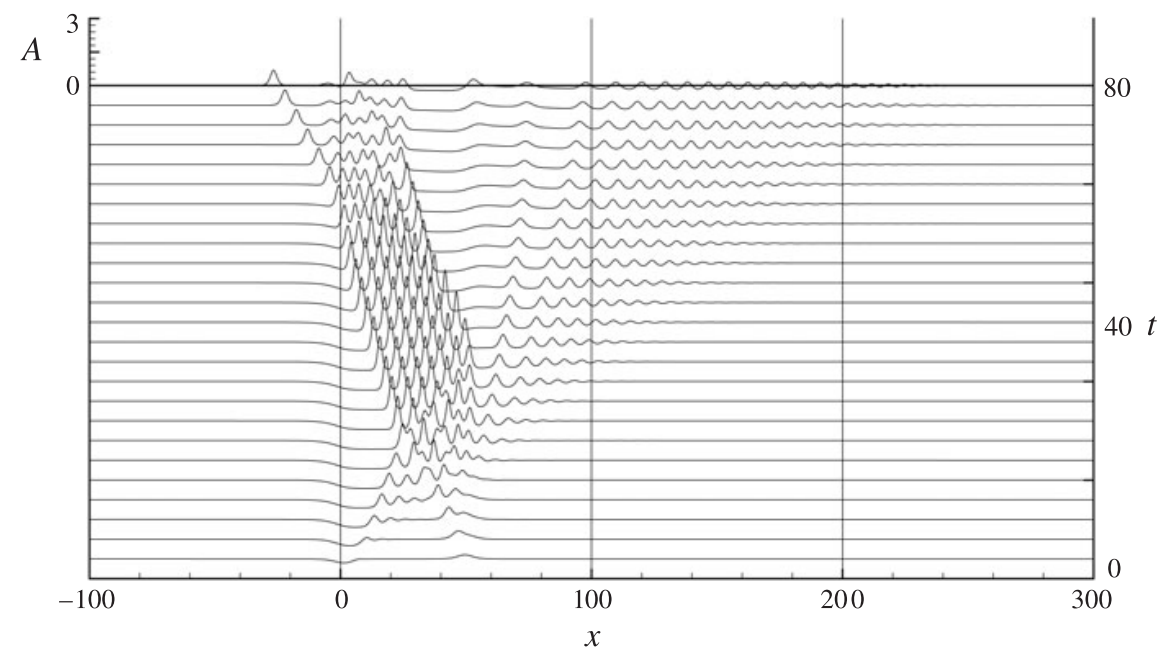

FIgURE 2. Numerical simulation of the fKdV equation (1.1) for a wide hole of width $W=50$ with $F_{M}=-0.45$ and $\Delta=0.0$.

unsteady over the obstacle, and this modulates the undular bores propagating away from the obstacle. A numerical simulation for a wide obstacle with negative polarity (a hole) is shown in Figure 2.

A single step is defined by

$$
F(x)=0, \quad x<0 \quad \text { and } \quad F(x)=F_{M}, \quad x>W,
$$

while $F(x)$ varies monotonically in $0<x<W$. A positive (negative) step has $F_{M}>0$ $\left(F_{M}<0\right)$. Strictly, $F(x)$ should return to zero for some $L \gg W$ in order to ensure conservation of mass in the $\mathrm{KdV}$ equation (1.1). However, our concern here is with the locally steady solution in the vicinity of the step, and so in this section we shall ignore this, and in effect assume that $L \rightarrow \infty$. In practice this means that the solutions constructed below are only valid for some limited time, determined by how long it takes for a disturbance to travel the distance $L$.

Grimshaw et al. [19, 20] extended the "hydraulic" approximation used in [18, 30], and described above in Section 2.1, to construct asymptotic solutions over the step (2.10). First, we construct the local steady-state solution $A_{s}(x)$ in the forcing region, which again satisfies (2.1), (2.2). However, expressions (2.3) are now replaced by

$$
C=-\Delta A_{-}+3 A_{-}^{2}=-\Delta A_{+}+3 A_{+}^{2}+F_{M} .
$$

Then, as in Section 2.1, $C$ is determined by considering the long-time limit of the forced Hopf equation with the initial condition $A(x, 0)=0$. The outcome for a positive step $\left(F_{M}>0\right)$ is that $C=F_{M}, F_{M}-\Delta^{2} / 12,0$ according as $\Delta<0,0 \leq \Delta<\left(12 F_{M}\right)^{1 / 2}$, $\Delta>\left(12 F_{M}\right)^{1 / 2}$. Then the corresponding solutions for $A_{ \pm}$are given by 


$$
\begin{aligned}
\Delta \leq 0: & 6 A_{-}=\Delta+\left(\Delta^{2}+12 F_{M}\right)^{1 / 2}, \quad 6 A_{+}=0 \\
0<\Delta<\left(12 F_{M}\right)^{1 / 2}: & 6 A_{-}=\Delta+\left(12 F_{M}\right)^{1 / 2}, \quad 6 A_{+}=\Delta, \\
\Delta>\left(12 F_{M}\right)^{1 / 2}: & 6 A_{-}=0, \quad 6 A_{+}=\Delta-\left(\Delta^{2}-12 F_{M}\right)^{1 / 2} .
\end{aligned}
$$

In the first two cases (2.12), (2.13), the upstream solution is positive $\left(A_{-}>0\right)$, while in the third case (2.14), $A_{-}=0$. Hence in the first two cases an undular bore is needed to describe the transition, and is given by (B.3)-(B.6) with $A_{0}=A_{-}$. In the third case, no transition is needed. But we now need to impose the extra requirement that the undular bore must lie in $x<0$. This leads to the condition that for a fully detached upstream undular bore,

$$
\Delta<-2\left(F_{M}\right)^{1 / 2}<0
$$

or if

$$
-2\left(F_{M}\right)^{1 / 2}<\Delta<\left(12 F_{M}\right)^{1 / 2},
$$

then the upstream undular bore is only partially formed and is attached to the obstacle. Downstream, in the first case (2.12), $A_{+}=0$ and no transition is needed. In the cases (2.13), (2.14), $A_{+}>0$ and the transition is through a rarefraction wave.

For a negative step, $F_{M}<0$, the outcome is

$$
\begin{aligned}
\Delta \geq 0: & 6 A_{-}=0, \quad 6 A_{+}=\Delta-\left(\Delta^{2}-12 F_{M}\right)^{1 / 2}, \\
-\left(-12 F_{M}\right)^{1 / 2}<\Delta<0: & 6 A_{-}=\Delta, \quad 6 A_{+}=\Delta-\left(-12 F_{M}\right)^{1 / 2}, \\
\Delta<-\left(-12 F_{M}\right)^{1 / 2}: & 6 A_{-}=\Delta+\left(\Delta^{2}+12 F_{M}\right)^{1 / 2}, \quad 6 A_{+}=0 .
\end{aligned}
$$

Here the constant in (2.11) is $C=0,-\Delta^{2} / 12, F_{M}$, respectively. In the first two cases (2.17), (2.18), the downstream solution is positive, $A_{+}>0$, and so the transition is through an undular bore. In the third case (2.19), $A_{+}=0$ and no transition is needed. Imposing the condition that the bore can only exist in $W>0$, we find that there is a fully detached downstream undular bore when

$$
\Delta>-\left(-3 F_{M}\right)^{1 / 2},
$$

or if

$$
-\left(-12 F_{M}\right)^{1 / 2}<\Delta<-\left(-3 F_{M}\right)^{1 / 2},
$$

then the downstream undular bore is only partially formed and is attached to the obstacle. For $\Delta<-\left(-12 F_{M}\right)^{1 / 2}$ we expect a stationary lee-wave train to form downstream. Upstream, in the first case (2.17) when $\Delta>0, A_{-}=0$ and no transition is needed, while in the last two cases (2.18), (2.19) when $\Delta<0, A_{-}<0$ and the transition is through a rarefraction wave.

\section{Steady forced solutions}

As described above in Section 2, the key quantities needed in this asymptotic approach are the limits $A_{ \pm}$(see (2.2)) of the locally steady solution $A_{s}(x)$ given by (2.1). While the "hydraulic" approximation is useful in that it leads to simple explicit 
expressions for $A_{ \pm}$, and has been found to agree quite well with numerical simulations, it provides no information on how the shape and width $L$ of the obstacle might affect the solution. Hence, in this section we construct some explicit solutions of (2.1) using a phase-plane analysis, for certain special choices of $F(x)$, where $F(x)=0, x<0, x>L$ and in $0<x<L$ either $F(x)>0$ for a positive obstacle, or $F(x)<0$ for a negative obstacle (a hole). The key parameters of interest are then the width $L$ and the largest height $F_{M}$, which is either positive or negative. Steady solutions for flow over an obstacle, or a step, have been considered by Dias and Vanden-Broeck [6-9], Binder et al. [2, 3] and Ee et al. [10, 12], using both numerical solutions and phase-plane analysis. Here we review and extend the recent study of Ee et al. [12], who examined steady solutions for wide obstacles and showed that, while for a positive obstacle only monotonic solutions could be found for which $A_{s}$ decreases from $A_{-}>0$ upstream to $A_{+}<0$ downstream, for a negative obstacle of sufficiently large width solutions for $A_{s}$ could be found with several trapped waves over the obstacle.

First we note that $A_{ \pm}$are the critical points of (2.1) in the $A_{s x}-A_{s}$ phase plane, when $F(x)=0$, that is, both upstream and downstream outside the forcing region. They are given by (2.3), so that

$$
6 A_{+}=\Delta-\left(\Delta^{2}+12 C\right)^{1 / 2}, \quad 6 A_{-}=\Delta+\left(\Delta^{2}+12 C\right)^{1 / 2} .
$$

Here the respective signs have been chosen to agree with the "hydraulic" approximation, and the $A_{ \pm}$exist for all allowed values of the constant $C$, that is, $\Delta^{2}+12 C>0$ where $C$ is yet to be determined. Then $A_{+}$is a saddle point, and $A_{-}$ is a centre. Since we require that $A_{+}<0<A_{-}$, and from (2.3) it can be shown that $C=-3 A_{+} A_{-}$, we see that $C>0$, that is, $3 A_{+}<\Delta<3 A_{-}$. A typical phase plane when $F(x)=0$ is shown in Figure 3, where we note especially the homoclinic orbit (dashed curve), and that on the periodic orbits the direction for $x$ increasing is clockwise. Then, in the region $0<x<L$ where $F(x) \neq 0$, the phase-plane orbits are modified as $F(x)$ varies, and our task is to use this modification to connect the upstream centre to the downstream saddle point. In Section 2.1 the "hydraulic" approximation was used to construct this connection. Here we shall instead use a piecewise constant forcing, for which $F(x)=F_{m}, 0<x<L$.

3.1. Narrow obstacle First we consider the special case where $F(x)=\Gamma \delta(x)$, which can be interpreted as the limit of the piecewise constant forcing as $L \rightarrow 0$ with $\Gamma=F_{M} L$ fixed. Then in equation (2.1) $F(x)$ is zero except at $x=0$, where the equation is replaced by the jump conditions

$$
\left[A_{s x}\right]_{0-}^{0+}=-\Gamma, \quad\left[A_{s}\right]_{0-}^{0+}=0 .
$$

The solution for $x \neq 0$ is described by the phase plane shown in Figure 3, so that for $x<0$ the solution is the centre $A_{s}=A_{-}$and for $x>0$ it is the homoclinic orbit through the saddle point at $A_{s}=A_{+}$. These are connected by the jump conditions (3.2) so that the starting point on the homoclinic orbit is given by $A_{s}(0+)=A_{+}, A_{s x}(0+)=-\Gamma$. In the phase plane shown in Figure 3, the connection is a vertical line from $A_{s x}=0$ 


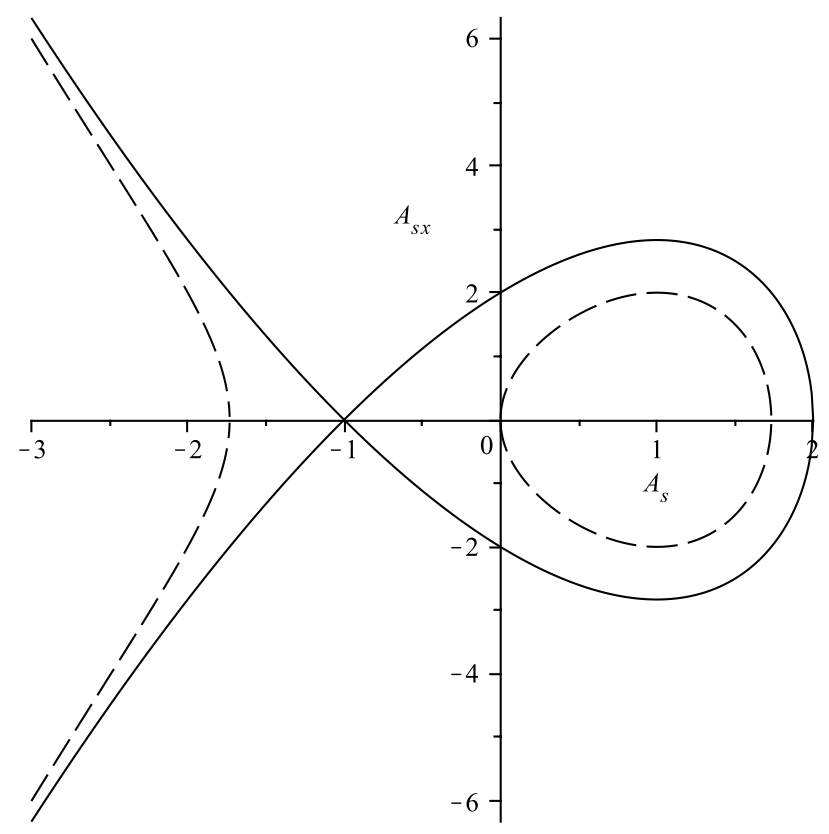

Figure 3. Phase plane for (2.1) when $F(x)=0, C=3, \Delta=0$.

to $A_{s x}=-\Gamma$, with $A_{s}=A_{-}$fixed. However, since $\Gamma$ is fixed, we must adjust the constant $C$ to match this jump, and so then determine $A_{ \pm}$from (2.3). The outcome is that

$$
\Gamma^{2}=\left(A_{-}-A_{+}\right)^{3} \text {. }
$$

Together with the last expression in (2.3) we have two equations for $A_{ \pm}$, and so we find that

$$
A_{ \pm}=\frac{\Delta}{6} \mp \frac{|\Gamma|^{2 / 3}}{2} \text {. }
$$

Further, since we require that $A_{-}>0>A_{+}$, this expression also defines the transcritical regime

$$
|\Delta|<3|\Gamma|^{2 / 3} \text {. }
$$

The homoclinic orbit through $A_{+}$is the stationary solitary wave

$$
A_{s}(x)=A_{s w}(x)=A_{+}+2 K^{2} \operatorname{sech}^{2}\left(K\left(x-x_{1}\right)\right), \quad 3\left(A_{-}-A_{+}\right)=4 K^{2} .
$$

The explicit solution is thus $A_{s}=0, x<0$ and $A_{s}=A_{s w}, x>0$, where the phase $x_{1}$ is given by

$$
A_{-}-A_{+}=2 K^{2} \operatorname{sech}^{2}\left(K x_{1}\right), \quad \Gamma=-4 K^{3} \operatorname{sech}^{2}\left(K x_{1}\right) \tanh \left(K x_{1}\right) .
$$

Elimination of $x_{1}$ recovers the expression (3.3). Note that this solution exists for both positive $(\Gamma>0)$ and negative $(\Gamma<0)$ obstacles, where $x_{1}<0$ for a positive obstacle and $x_{1}>0$ for a negative obstacle, a result which can also be directly inferred from the 
phase plane. Thus for a positive obstacle, only a suppressed solitary wave is formed, since only a portion of the region where $A_{s w}$ is monotonically decreasing as $x$ increases is needed. But for a negative obstacle, the solitary wave crest at $x=x_{1}$ is part of the solution, and the downstream flow is wave-like. This feature of the flow over a negative obstacle will appear below in a stronger form for wide obstacles.

3.2. Wide positive obstacle Next we consider the solution for the piecewise constant forcing of constant width, that is, $F(x)=F_{M}, 0<x<L$. As above, the solution outside the forcing region is described by the phase plane shown in Figure 3, so that for $x<0$ the solution is the centre $A_{s}=A_{-}$and for $x>L$ it is the homoclinic orbit, that is, the solitary wave $A_{s w}(x)$ (see (3.6)) through the saddle point at $A_{s}=A_{+}$. These are to be connected by an orbit from the phase plane in the forcing region, obtained from (2.1) with $F(x)=F_{M}$. The boundary conditions at the ends of the forcing region are the continuity of $A_{s x}, A_{s}$ at $x=0, L$. Hence we set the upstream boundary condition in the forcing region as

$$
A_{s}(0+)=A_{-}, \quad A_{s x}(0+)=0 .
$$

If $A_{-}$is known, these conditions determine a unique solution as a function of the parameters $\Delta, F_{M}, L$. The downstream solution in $x>L$ is the homoclinic orbit, that is, the solitary wave (3.6), and so the matching conditions as $x \rightarrow L-$ are given by

$$
\begin{gathered}
A_{s}(L-)=A_{+}+2 K^{2} \operatorname{sech}^{2}\left(K\left(L-x_{1}\right)\right), \\
A_{s x}(L-)=-4 K^{3} \operatorname{sech}^{2}\left(K\left(L-x_{1}\right)\right) \tanh \left(K\left(L-x_{1}\right)\right) .
\end{gathered}
$$

These generate two equations for $x_{1}$, and hence the constant $C$ in (2.1) must be adjusted to ensure that a solution exists. Elimination of $x_{1}$ from (3.8), (3.9) yields a single expression connecting $A_{s x}(L-)$ and $A_{s}(L-)$,

$$
A_{s x}^{2}(L-)=\left(A_{s}(L-)-A_{+}\right)^{2}\left\{3\left(A_{-}-A_{+}\right)-2\left(A_{s}(L-)-A_{+}\right)\right\},
$$

which provides the required third equation in addition to (2.3) for the unknowns $A_{ \pm}, C$. In the phase plane for the forcing region, the upstream conditions (3.7) define a unique orbit through the centre at $A_{s}=A_{-}$, and the construction described above is interpreted as the requirement that this orbit intersects the homoclinic orbit through $A=A_{+}$with an intercept of total length $L$.

First we suppose that $F_{M}>0$, corresponding to a positive obstacle. The critical points in the forcing region are given by (3.1) with $C$ replaced by $C-F_{M}$. They exist only when $\Delta^{2}+12 C>12 F_{M}$, equivalent to $\left(\Delta-6 A_{ \pm}\right)^{2}>12 F_{M}$ on using (2.3), and then consist of a saddle point at $A_{s}=A_{1}$ and a centre at $A_{s}=A_{2}$, where $A_{+}<A_{1}<A_{2}<A_{-}$. If $\Delta^{2}+12 C>16 F_{M}$, that is, $\left(\Delta-6 A_{ \pm}\right)^{2}>16 F_{M}$, then in the forcing region phase plane, the homoclinic orbit through the saddle point where $A_{s}=A_{1}$ contains the critical point $A_{s}=A_{-}$, and so the required orbit through $A_{s}=A_{1}$ is periodic and is contained within this homoclinic orbit. This in turn lies inside the homoclinic orbit $A_{s w}(x)$ (see (3.6)) through $A_{s}=A_{+}$in the phase plane outside the 


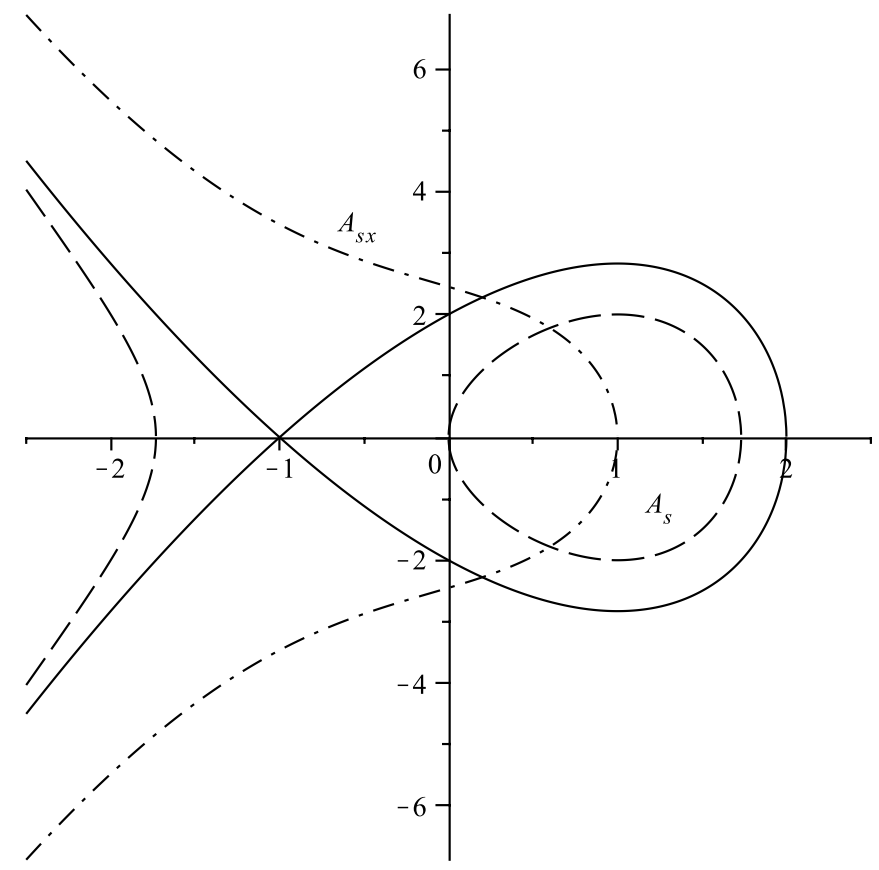

Figure 4. Phase plane for (2.1) when $C=3, \Delta=0, A_{ \pm}=\mp 1, F_{M}=5$.

forcing region. Hence there can be no intersections, and hence no steady solution. But if instead $12 F_{M}<\Delta^{2}+12 C<16 F_{M}$, that is, $12 F_{M}<\left(\Delta-6 A_{ \pm}\right)^{2}<16 F_{M}$, then the critical point $A_{s}=A_{-}$lies outside this forcing region homoclinic orbit, and so the required orbit through $A_{s}=A_{-}$will make an intercept. Finally, if $\Delta^{2}+12 C<12 F_{M}$, that is, $\left(\Delta-6 A_{ \pm}\right)^{2}<12 F_{M}$, then there are no critical points in the forcing region, and again an intercept can be achieved. A typical example is shown in Figure 4, where the required orbit in the forcing region is the dash-dotted curve, and the other orbits are those for the phase plane outside the forcing region, as shown in Figure 3. We see that the required orbit necessarily has $A_{s x}(x)<0, A_{s}(x)<A_{-}$, and so the connecting orbit describes a monotonic decrease of $A_{s}$ from $A_{-}$upstream to $A_{+}$downstream. Further, the intercept with the homoclinic orbit $A_{s w}(x)$ (see (3.6)) always generates only a suppressed solitary wave.

It remains to determine the constant $C$ in the allowed case when $\Delta^{2}+12 C<16 F_{M}$. From the phase-plane analysis described above, we must use an unbounded orbit of (2.1) in the forcing region through the critical point $A_{s}=A_{-}$, and then apply the matching conditions (3.10). Thus it is readily found that this orbit intersects the solitary wave orbit when $A_{s}=A_{i}$, where

$$
2 F_{M}\left(A_{-}-A_{i}\right)=\left(A_{-}-A_{+}\right)^{3}
$$


and then the required length $L$ is given by

$$
L=\int_{A_{i}}^{A_{-}} \frac{d A}{\left\{\left(A_{-}-A\right)^{2}\left(\Delta-4 A_{-}-2 A\right)+2 F_{M}\left(A_{-}-A\right)\right\}^{1 / 2}} .
$$

Expression (3.12) is the required third condition, which when combined with (2.3) determines $A_{ \pm}, C$ in terms of the parameters $\Delta, F_{M}, L$. However, the solution is constrained by the need to impose the condition that $C>0$, that is, $3 A_{+}<\Delta<$ $3 A_{-}$. Further analysis is assisted by changing variables in (3.11), (3.12) by setting $B=\left(A_{-}-A\right) /\left(A_{-}-A_{+}\right)$, so that

$$
L A_{d}^{1 / 2}=\int_{0}^{B_{i}} \frac{d B}{\left\{2 B^{3}-3 B^{2}+B / B_{i}\right\}^{1 / 2}},
$$

where

$$
2 F_{M} B_{i}=A_{d}^{2} \text { and } A_{d}=A_{-}-A_{+} .
$$

Expression (3.13) defines $A_{d}$ (or equivalently $B_{i}$ ) in terms of $F_{M}, L$ and hence determines the required steady solution. In principle the allowed range for $B_{i}$ is $0<B_{i}<1$, but the cubic polynomial for $B$ in the denominator of (3.13) has a double zero at $B=3 / 4$ when $B_{i}=8 / 9$, and so the actual allowed range is $0<B_{i}<8 / 9$. In the limit $B_{i} \rightarrow 0$, (3.13) reduces to $L F_{M} \rightarrow A_{d}^{3 / 2}$ which is just the narrow obstacle limit (see (3.3), (3.4)). On the other hand, as $B_{i} \rightarrow 8 / 9$, (3.13) implies that $L \rightarrow \infty$, $16 F_{M}=9 A_{d}^{2}$, and so

$$
6 A_{ \pm}=\Delta \mp 4 F_{M}^{1 / 2},
$$

while the transcritical regime is then given by

$$
|\Delta|<4 F_{M}^{1 / 2}
$$

Note that this limit corresponds to the limit when $\Delta^{2}+12 C \rightarrow 16 F_{M}$ from above, and hence in the phase plane the required orbit through $A_{s}=A_{-}$lies just outside the homoclinic orbit through $A_{s}=A_{1}$. Curiously, although these expressions (3.15), (3.16) have the same scaling as the corresponding solutions in the "hydraulic" approximation (2.4), (2.5), there is a different numerical factor, in that effectively $12 F_{M}$ in the "hydraulic" approximation is here replaced by $16 F_{M}$. This is presumably because the sharp ends here at $x=0, L$ violate the assumptions of the "hydraulic" approximation. Otherwise, in general it can be shown from (3.13) that $L A_{d}^{1 / 2}$ is a monotonically increasing function of $B_{i}$, and hence for every fixed $L, 0<L<\infty$ and fixed $F_{M}>0$, we can deduce that there is a unique solution for $A_{d}$ in the allowed range, and hence a unique solution for $A_{ \pm}$. As $L$ increases, so does $A_{d}$, and for each fixed $L, A_{d}$ increases as $F_{M}$ increases. These explicit results for a piecewise constant forcing are in broad agreement with the numerical results of Ee et al. [10, 12] for a smooth forcing term. Two plots of (3.13) are shown in Figure 5 for $F_{M}=1,4$ together with the corresponding limiting approximations as $L \rightarrow 0, \infty$. Note that the combination of these two limits provides a close approximation to the whole curve for nearly all $L$, as found by Grimshaw and Smyth [18], Smyth [30] and Ee et al. [10, 12]. 


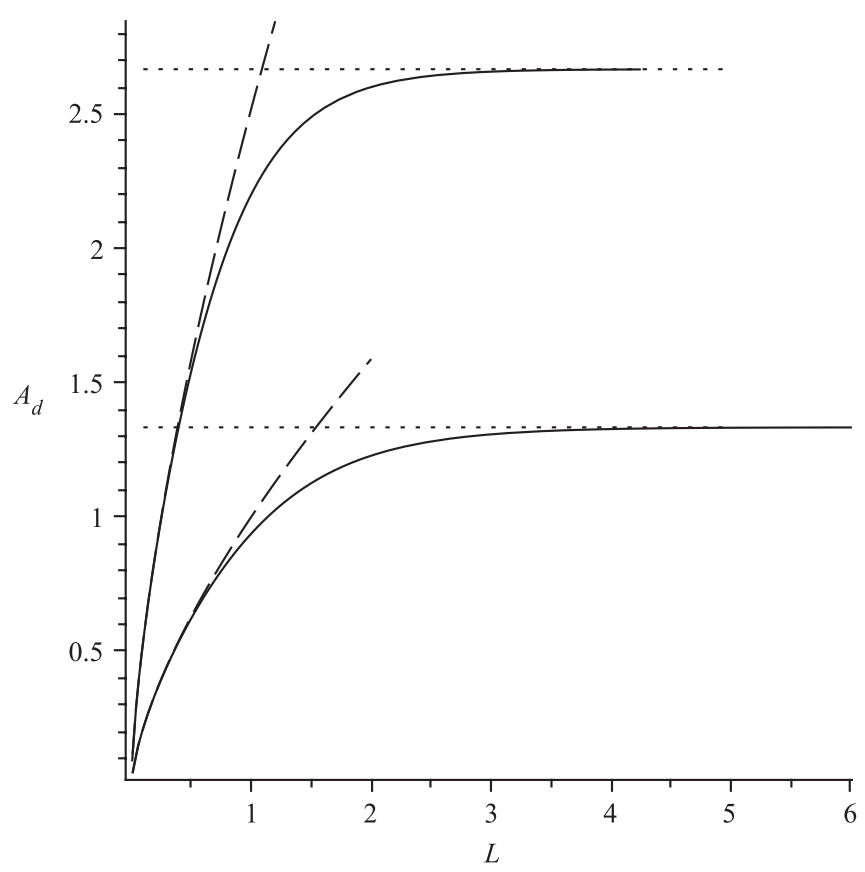

FIGURE 5. The solution of (3.13) for $A_{d}$ as a function of $L$, for $F_{M}=1,4$ (lower solid curve, upper solid curve), together with the corresponding limiting approximations as $L \rightarrow 0, \infty$ (dashed curve, dotted line).

3.3. Wide negative obstacle Here we suppose that $F_{M}<0$, corresponding to a negative obstacle. The critical points in the forcing region are again given by (3.1) with $C$ replaced by $C-F_{M}$. They exist only when $\Delta^{2}+12 C>12 F_{M}$, but now since $C>0, F_{M}<0$, we see that they exist for all parameter values, and consist of a saddle point at $A_{s}=A_{1}$ and a centre at $A_{s}=A_{2}$, where $A_{1}<A_{+}<0<A_{-}<A_{2}$. It can now be shown that the homoclinic orbit in the forcing region through $A_{s}=A_{1}$ encloses the homoclinic orbit $A_{s w}(x)$ (see (3.6)) outside the forcing region through $A_{s}=A_{+}$. Hence the required connecting orbit in the forcing region through $A_{s}=A_{-}$must be a periodic orbit around the centre at $A_{s}=A_{2}$. A typical scenario is shown in Figure 6, where the required orbit in the forcing region is the dash-dotted curve, and the other orbits are those for the phase plane outside the forcing region, as shown in Figure 3. Note that under the present constraints $\left(A_{-}>0>A_{+}\right)$it can be shown that the required intercept always exists. As before, the constant $C$ must then be adjusted so that the connecting orbit has length $L$. Because the connecting orbit is periodic, the corresponding solution in the forcing region is wave-like. Further, as $L$ increases it will become necessary to move clockwise around this orbit several times. Thus the first intercept, which lies in $A_{s x}>0, A_{s}>A_{-}$, describes a partial periodic wave with the crest excluded, followed by a solitary wave whose crest at $x=x_{1}$ is part of the solution and where $A_{s x}=0$; for $x>x_{1}, A_{s x}(x)<0$. The next possible intercept as $L$ increases lies in $A_{s x}<0, A_{s}>A_{-}$ 


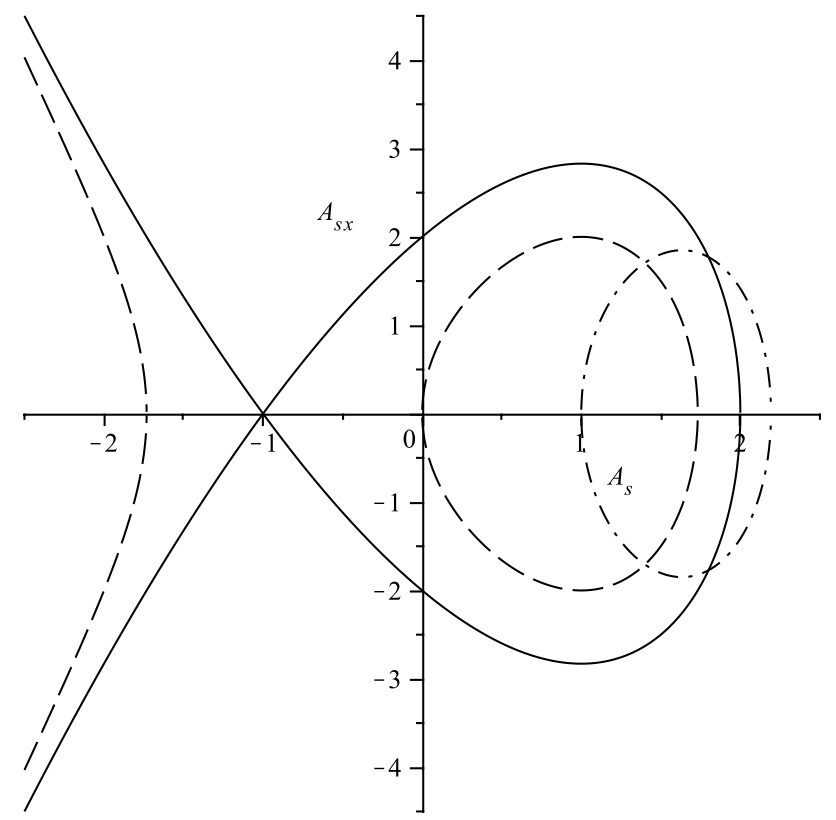

FIGURE 6. Phase plane for (2.1) when $C=3, \Delta=0, F_{M}=-5$.

and describes a periodic wave whose crest is included, connecting to a suppressed solitary wave. As $L$ increases further, this pattern is repeated, and importantly each complete circuit of the periodic orbit corresponds to one complete wavelength of a periodic wave in the forcing region. Thus in general the solution $A_{s}(x)$ in the forcing region consists of $N$ periodic waves, followed by either a partial periodic wave with the crest excluded connecting in $x>L$ to a solitary wave $A_{s w}(x)$ with its crest included, or by a partial periodic wave with the crest included connecting in $x>L$ to a suppressed solitary wave $A_{s w}(x)$ whose crest is excluded. We note that an analogous phaseplane argument was used by Dias and Vanden-Broeck [9] to construct trapped waves between two widely separated localized positive obstacles, confirming their numerical solutions of the full steady Euler equations.

This phase-plane analysis can be done explicitly, since the periodic orbit in the forcing region $0<x<L$ can be represented explicitly by a cnoidal wave solution of (2.1). Thus we put $B=\left(A-A_{-}\right) / A_{d}, A_{d}=A_{-}-A_{+}$, and then the required orbit satisfies

$$
\frac{B_{x}^{2}}{A_{d}}=-2 B^{3}-3 B^{2}+\frac{B}{B_{i}}, \quad 2 F_{M} B_{i}=-A_{d}^{2} .
$$

Note the change of signs here in the definition of $B, B_{i}$ compared to that in (3.14), since we expect $B(x)>0$. Also we have applied the upstream matching condition (3.7) that $B_{x}(0)=B(0)=0$. Note that as above in Section 3.2, $B=B_{i}$ defines the intersection of this cnoidal wave orbit with the solitary wave orbit (3.6). The cnoidal wave solution 
of (3.17) that satisfies the boundary condition (3.7) at $x=0$ is (see Ee et al. [12])

$$
B(x)=a \mathrm{cn}^{2}\left(\beta\left(x-x_{0}\right) ; m\right), \quad \text { where } \beta x_{0}=K(m)
$$

and

$$
\beta^{2}=\frac{3 A_{d}}{4(1-2 m)}, \quad a=\frac{3 m}{2(1-2 m)}, \quad B_{i}=\frac{2(1-2 m)^{2}}{9 m(1-m)} .
$$

Here $\operatorname{cn}(u ; m)$ is the Jacobi elliptic function of modulus $m, 0<m<1$, with the spatial period $4 K(m) / \beta$, and $K(m)$ is the elliptic integral of the first kind. Note that since $\beta^{2}>0,0<m<1 / 2$ and $a>0$. Finally, we impose the condition that $B(L)=B_{i}$, so that

$$
a \mathrm{cn}^{2}(\beta L-K(m) ; m)=B_{i} .
$$

This is the required condition which determines the constants $C, A_{ \pm}$in terms of $F_{M}, \Delta, L$.

Then, using the formulas in (3.19), (3.20) can be regarded as an equation defining $\beta L=G(m)$ given by

$$
\frac{27 m^{2}(1-m)}{4(1-2 m)^{3}} \mathrm{cn}^{2}(G(m)-K(m) ; m)=1 .
$$

Further, since $0 \leq \mathrm{cn}^{2}(\cdot) \leq 1$, the expression (3.21) can only have solutions in the range $1 / 5<m<1 / 2$. Hence, from (3.19), $1 / 2<a<\infty$ and $1 / 2>B_{i}>0$, while elimination of $\beta^{2}$ yields

$$
-F_{M} L^{4}=4 G(m)^{4} m(1-m), \quad 3 A_{d} L^{2}=4 G(m)^{2}(1-2 m),
$$

determining $A_{d}$ from $F_{M}, L$ in terms of the parameter $m$. Importantly, $G(m)$ is multivalued, since we can write $G(m)=2 n K(m) \pm G_{0}(m), n=0,1,2, \ldots$, where $G_{0}(m)$ is such that $0<G_{0}(m)<K(m)$. The integer $n$ determines the number of trapped wave crests in $0<x<L$, and the alternate signs \pm correspond to $B_{x}(L)>0, B_{x}(L)<0$, corresponding to partial solitary waves with crests or to suppressed solitary waves, respectively, in $x>L$. A plot of $G_{0}(m)$ is shown in Figure 7 . Note that $G_{0}(m)$ increases from 0 to 1.68 as $m$ decreases from $1 / 2$ to $1 / 5$, that is, as $B_{i}$ increases from 0 to 1/2. As $L \rightarrow 0, m \rightarrow 1 / 2, B_{i} \rightarrow 0$ and $L F_{M} \rightarrow A_{d}^{3 / 2}$, which is the narrow obstacle limit (3.3), (3.4) of Section 3.1. A plot of the solution of (3.22) is shown in Figure 8 for the upper branch when $G(m)=G_{0}(m), n=0$, corresponding to Figure 7. Finally, in Figure 9 we show the first four branches for $A_{d}$ as a function of $L$ for two fixed values of $F_{M}$. Each plot exhibits two loops as $L$ increases, and as more branches are added, more loops will appear. On each loop with a fixed $F_{M}<0, A_{d}$ has a maximum value $\left|F_{M}\right|^{1 / 2}$ achieved when $L=2\left(2 n K(1 / 5)+G_{0}(1 / 5)\right)\left|25 F_{M}\right|^{-1 / 4}$. Note that the loops meet at the sequence of points $L=L_{n}=2 n K(1 / 2) /\left(-F_{M}\right)^{1 / 4}$. As $L \rightarrow L_{n}, A_{d} \rightarrow 0$, implying that then $A_{ \pm} \rightarrow 0, \Delta \rightarrow 0$, while the wave amplitude $a \rightarrow \infty$. Hence there is no transcritical regime for these special values of $L=L_{n}$, where it seems that instead a nonlinear resonance will occur. All the results described above agree qualitatively with the numerical solutions of Ee et al. [12] for a smooth wide negative obstacle. 


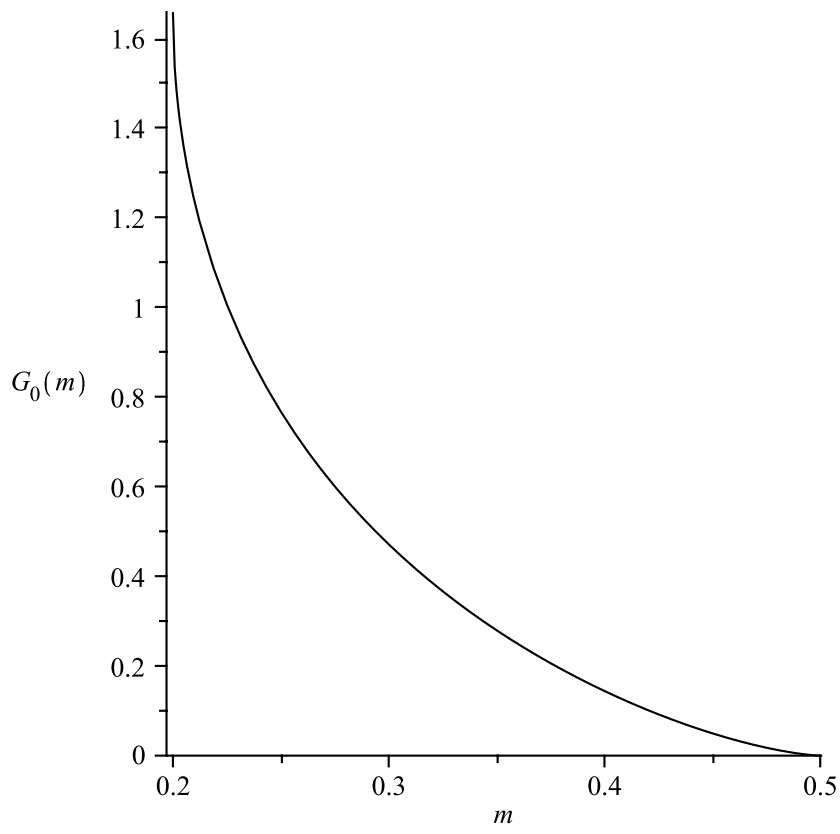

Figure 7. Plot of $G_{0}(m)$.

\section{Discussion}

In this paper we have reviewed the asymptotic solution of the fKdV equation (1.1) proposed by Grimshaw and Smyth [18] and Smyth [30] for flow over a localized obstacle and recently extended by Grimshaw et al. $[19,20]$ to flow over a step. The essential hypothesis is that the solution consists of upstream and downstream undular bores connected by a locally steady solution over the obstacle. The role of this locally steady solution is to provide the downstream and upstream constant elevations $A_{ \pm}$ with $A_{+}<0<A_{-}$, so that the undular bore solutions can then be constructed using modulation theory, as described in Appendix B. Hence we have in Section 3 focused especially on the structure of this locally steady solution, with an emphasis on the obstacle width as well as its amplitude, following the approach used in the studies by Ee and Clarke [10] and Ee et al. [12]. However, whereas these works used primarily numerical solutions to find the allowed steady states, here we have examined the special case of a piecewise constant obstacle, for which a complete explicit solution can be found using phase-plane methods.

For a positive obstacle, our explicit analysis confirms several previous numerical solutions of both the unsteady fKdV equation (1.1) and the steady fKdV equation (2.1), which show that a locally steady solution of the required kind, decreasing monotonically from $A_{-}>0$ upstream to $A_{+}<0$ downstream, can always be found for all obstacle heights and widths. Hence the interpretation of the upstream and 


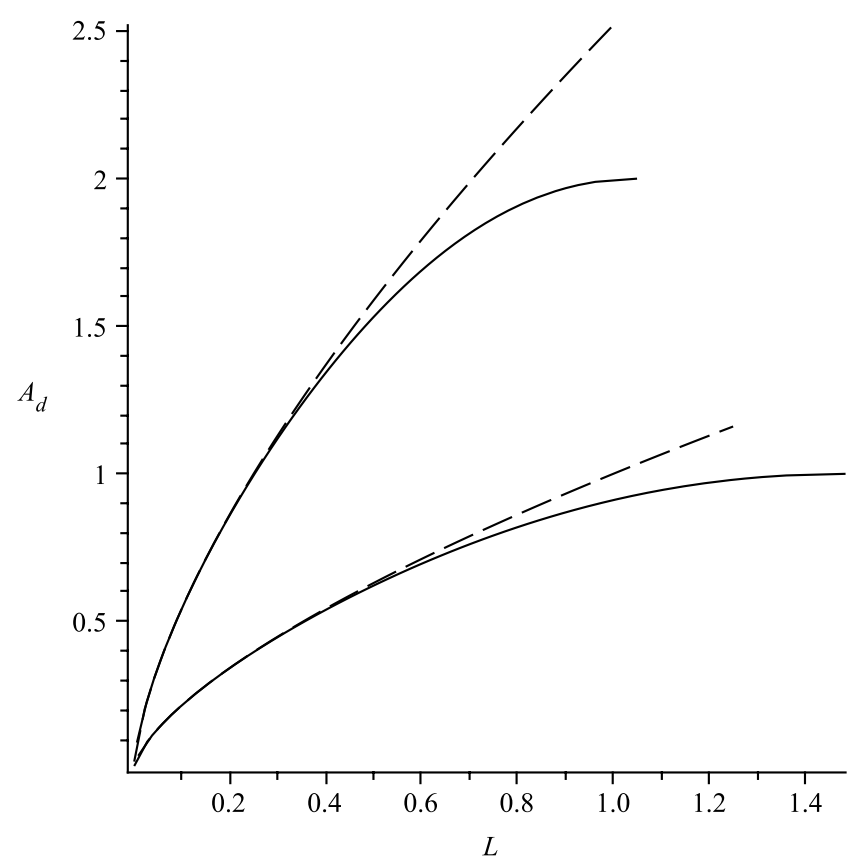

FIGURE 8. The solution of (3.22) for the upper branch when $n=0$ for $A_{d}$ as a function of $L$, for $F_{M}=-1,-4$ (lower solid curve, upper solid curve), together with the corresponding limiting approximation as $L \rightarrow 0$ (dashed curve).

downstream solutions outside the obstacle as undular bores propagating away from the obstacle would seem to be valid for all positive obstacles, independently of the obstacle width. However, in agreement with the recent study by Ee et al. [12], we find from our explicit analysis for a negative obstacle that while such required steady solutions can indeed be found for all obstacle heights and widths, the solution is not a monotonic transition from $A_{-}>0$ upstream to $A_{+}<0$ downstream, and instead is characterized by the formation of trapped waves over the obstacle (in this case a hole), where the number of such waves increases as the width increases. Although it might now seem possible to construct downstream and upstream propagating undular bores as can be done for a positive obstacle, some caution is needed. Several numerical simulations of the unsteady fKdV equation (1.1) (see [18-20], for instance) seem to show that the solution remains unsteady over a negative obstacle for long times, with a consequent modulation of the downstream and upstream propagating undular bores. While it may simply be the case that the desired steady state over the obstacle requires a very long time to be reached, we explore alternative explanations as to why the steady state constructed for a negative obstacle may not be realizable from the zero initial condition used in the cited numerical simulations. First we note that Ee and Clarke [10, 11] showed that for a localized negative obstacle, these steady solutions were unstable, whereas the steady solutions constructed for a positive localized obstacle were stable. 


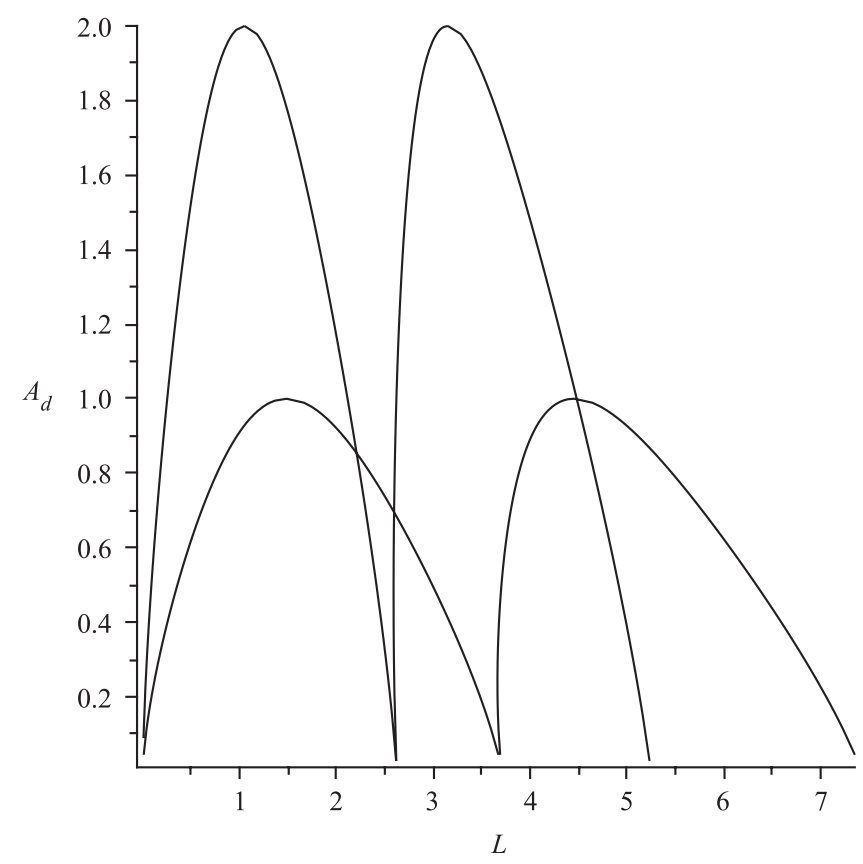

FIgURE 9. The solution of (3.22) for $A_{d}$ as a function of $L$, for $F_{M}=-1,-4$ (lower curve, upper curve) for the first four branches: $n=0$ (upper branch), $n=1$ (both branches) and $n=2$ (upper branch).

Next, we note that the undular bore construction described in Section 2 inevitably leads to only a partial undular bore either upstream or downstream. Consequently, a cnoidal wave with a particular modulus and amplitude is always being generated at either the immediate upstream or downstream side of the obstacle. For a positive obstacle, this can be matched to the locally steady monotonic solution. But for a negative obstacle this needs to be matched to another cnoidal wave with, in general, a different modulus and amplitude, and this seems unlikely to be achievable.

\section{Appendix A. Derivation of the forced Korteweg-de Vries equation}

The fKdV equation (1.1) was derived by Grimshaw and Smyth [18] for transcritical flow of a density-stratified fluid over an obstacle, and extended by Clarke and Grimshaw [4] to the case when the oncoming flow is vertically sheared. Here we give a brief outline of a heuristic alternative derivation. We begin with a summary of the derivation of the Korteweg-de Vries $(\mathrm{KdV})$ equation for weakly nonlinear long waves propagating on a background of a density-stratified shear flow in the absence of any forcing term. This is now a well-known result (see the recent review by Grimshaw et al. [17] and the references therein).

Consider a two-dimensional configuration where $x, z$ are the spatial coordinates in the horizontal and vertical directions, respectively. In the basic state the fluid is 
confined between a free surface at $z=0$ and a rigid bottom at $z=-h$, and has a density field $\rho_{0}(z)$, a horizontal shear flow $u_{0}(z)$ in the $x$-direction, and a pressure field $p_{0}(z)$ such that $p_{0 z}=-g \rho_{0}$. The density stratification is described by the buoyancy frequency $N(z)$, where

$$
N^{2}(z)=-\frac{g \rho_{0 z}}{\rho_{0}} .
$$

Then, relative to this basic state, we construct an asymptotic expansion for weakly nonlinear long waves. For this purpose a small parameter $\epsilon \ll 1$ is introduced. At the leading order we get linear long wave theory,

$$
\zeta \sim \epsilon^{2} A(X, T) \phi(z)+\cdots, \quad X=\epsilon(x-c t), T=\epsilon^{3} t .
$$

Here $\zeta$ is the vertical particle displacement relative to the basic state, and the modal function $\phi(z)$ satisfies the system

$$
\begin{aligned}
& \left\{\rho_{0}\left(c-u_{0}\right)^{2} \phi_{z}\right\}_{z}+\rho_{0} N^{2} \phi=0 \text { for }-h<z<0, \\
& \phi=0 \quad \text { at } z=-h, \quad\left(c-u_{0}\right)^{2} \phi_{z}=g \phi \quad \text { at } z=0 .
\end{aligned}
$$

Equation (A.3) is the long-wave limit of the Taylor-Goldstein equation, and with the boundary conditions (A.4) determines the modal function and the linear long wave speed $c$. Typically this boundary-value problem (A.3), (A.4) defines an infinite sequence of modes, but here we select the resonant mode defined by $c=0$, or allowing for a small detuning, we set $c=\epsilon^{2} \Delta$. Then, as time increases, the hitherto neglected nonlinear terms come into play and cause wave steepening. However, this is opposed by the terms representing linear wave dispersion, also neglected in the linear long wave theory. A balance between these effects emerges as time increases, technically obtained as a compatibility condition at the second order in the expansion. The outcome is the $\mathrm{KdV}$ equation for the wave amplitude,

$$
A_{T}+\mu A A_{X}+\delta A_{X X X}=0
$$

where

$$
I \mu=3 \int_{-h}^{0} \rho_{0}\left(c-u_{0}\right)^{2} \phi_{z}^{3} d z, \quad I \delta=\int_{-h}^{0} \rho_{0}\left(c-u_{0}\right)^{2} \phi^{2} d z
$$

and

$$
I=2 \int_{-h}^{0} \rho_{0}\left(c-u_{0}\right) \phi_{z}^{2} d z
$$

Setting $c=\epsilon^{2} \Delta$ and reverting to the original unscaled variables, equation (A.5) becomes

$$
-A_{t}-\Delta A_{x}+\mu_{0} A A_{x}+\delta_{0} A_{x x x}=0
$$

where

$$
I_{0} \mu_{0}=3 \int_{-h}^{0} \rho_{0} u_{0}^{2} \phi_{0 z}^{3} d z, \quad I_{0} \delta_{0}=\int_{-h}^{0} \rho_{0} u_{0}^{2} \phi_{0}^{2} d z
$$


and

$$
I_{0}=2 \int_{-h}^{0} \rho_{0} u_{0} \phi_{0 z}^{2} d z
$$

Here the modal function $\phi_{0}(z)$ is the solution of the modal equations (A.3), (A.4) with $c=0$.

The next step is to introduce the obstacle and hence add a forcing term to the KdV equation (A.7). Thus we now let the bottom boundary condition be given by

$$
w+\left(u_{0}+u\right) b_{x}=0 \quad \text { on } z=-h+b(x)
$$

where $u, w$ are the perturbed horizontal and vertical velocity fields and $b(x)$ represents the bottom obstacle. Since we assume that $b$ is $O\left(\epsilon^{4}\right)$, it is sufficient to consider the linearized boundary condition

$$
\zeta_{t}+u_{0} \zeta_{x}=-u_{0} b_{x} \quad \text { on } z=-h
$$

To find the effect of this boundary condition, it is sufficient to consider the linear long wave theory, given by

$$
\begin{gathered}
\left\{\rho_{0}\left(\frac{\partial}{\partial t}+u_{0} \frac{\partial}{\partial x}\right)^{2} \zeta_{z}\right\}_{z}+\rho_{0} N^{2} \frac{\partial^{2} \zeta}{\partial x^{2}}=0, \quad-h<z<0, \\
\left(\frac{\partial}{\partial t}+u_{0} \frac{\partial}{\partial x}\right)^{2} \zeta_{z}=-g \frac{\partial^{2} \zeta}{\partial x^{2}}, \quad z=0 .
\end{gathered}
$$

Note that the modal equation (A.4) is just (A.11) in Fourier space, while the boundary condition (A.4) likewise corresponds to the free-surface boundary condition (A.12). However, the bottom boundary condition in (A.4) is now replaced by the boundary condition (A.10), which has a forcing term on the right-hand side.

Next we impose the initial conditions that $\zeta=0, \zeta_{t}=0$ at $t=0$ and also assume that the obstacle is introduced at $t=0$. The solution of the system (A.10), (A.11), (A.12) can now be found using Fourier transforms and is given by

$$
\begin{gathered}
\zeta=\frac{1}{4 \pi^{2}} \int_{-\infty}^{\infty} \int_{-\infty}^{\infty} \hat{\zeta} \exp (i k x-i \omega t) d k d \omega \\
\hat{\zeta}=\frac{\phi(z ; c)}{\phi(-h ; c)} \frac{u_{0}(-h)}{u_{0}(-h)-c} \frac{\hat{b}}{i \omega} .
\end{gathered}
$$

Here $c=\omega / k$ and $\phi(z ; c)$ is the solution of the modal equation (A.3) and the free surface boundary condition in (A.4) for a given $c$. Note that the $\omega$-integral is displaced slightly into $\operatorname{Im} \omega>0$ to ensure causality. The solution is now found by replacing $\omega$ with $c=\omega / k$ and evaluating the resulting $c$-integral at the poles on the real $c$-axis. These poles are at $c=0$ and at the solutions of $\phi(-h ; c)=0$, which defines the modal functions and the corresponding linear long wave speeds. The solution thus consists of a steady term and a set of linear long waves. For the present purpose it is 
sufficient to consider only the resonant linear long wave with speed $c=\Delta$, when the outcome is

$$
\zeta=A(x, t) \phi_{0}(z), \quad A(x, t)=\frac{\gamma}{\Delta}\{b(x)-b(x-\Delta t)\},
$$

where

$$
I_{0} \gamma=\rho_{0} u_{0}^{2} \phi_{0 z} \quad \text { at } z=-h .
$$

But now we see that this solution solves the initial-value problem for the linear long wave equation

$$
-A_{t}-\Delta A_{x}+\gamma b_{x}(x)=0 .
$$

This result can now be compared with the unforced $\mathrm{KdV}$ equation (A.7) when the nonlinear and dispersive terms are omitted. Thus we infer that the forced $\mathrm{KdV}$ equation must have the form

$$
-A_{t}-\Delta A_{x}+\mu_{0} A A_{x}+\delta_{0} A_{x x x}+\gamma b_{x}(x)=0 .
$$

It is readily established that this agrees with the corresponding result of Clarke and Grimshaw [4], and also with that of Grimshaw and Smyth [18] for the special case when $u_{0}=U$ is a constant. Finally, we can rescale by

$$
\tilde{A}=\frac{\mu_{0} A}{6 \delta_{0}}, \quad \tilde{\Delta}=\frac{\Delta}{\delta_{0}}, \quad \tilde{t}=\delta_{0} t, \quad F=\frac{\gamma \mu_{0} b}{6 \delta_{0}^{2}},
$$

so that (A.18) adopts the canonical from (1.1). Note that it has been assumed here that $\delta_{0}>0$, which is the case whenever $u_{0}>0$. But if $\delta_{0}<0$, the further transformation $t \rightarrow-t, x \rightarrow-x$ can be used in (1.1) to restore the canonical form.

\section{Appendix B. Undular bore}

The term "undular bore" is widely used in the literature in a variety of contexts and with several different meanings. In general, an undular bore is an oscillatory transition between two different basic states. Here, since we are concerned only with nondissipative systems, an undular bore is intrinsically unsteady. A simple representation of an undular bore can be obtained from the solution of the unforced $\mathrm{KdV}$ equation

$$
-A_{t}-\Delta A_{x}+6 A A_{x}+A_{x x x}=0
$$

with the initial condition

$$
A=A_{0} H(x),
$$

where we assume at first that $A_{0}>0$. Here $H(x)$ is the Heaviside function (that is, $H(x)=1$ if $x>0$ and $H(x)=0$ if $x<0)$. The solution can in principle be obtained through the inverse scattering transform. However, it is more instructive to use the asymptotic method developed in $[22,32]$. In this approach, the solution of (B.1) with this initial condition is represented as the modulated periodic wave train

$$
A=a\left\{b(m)+\mathrm{cn}^{2}(\kappa(x-V t) ; m)\right\}+d,
$$




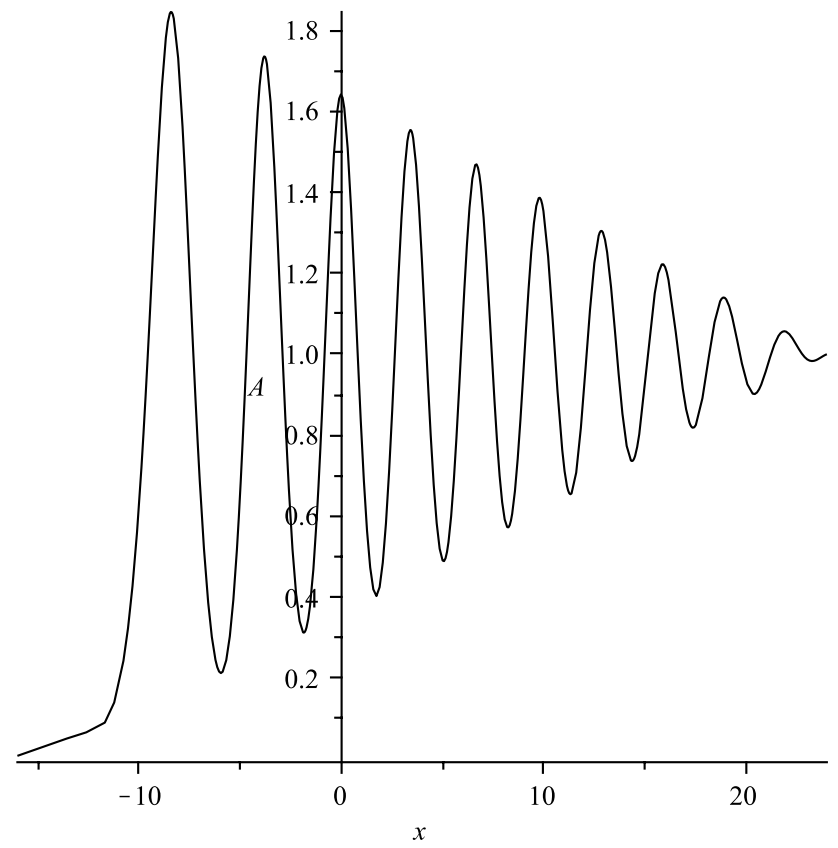

FIgure 10. A plot of the undular bore given by (B.5), (B.6) for $A_{0}=1, \Delta=0, t=4$.

where

$$
b=\frac{1-m}{m}-\frac{E(m)}{m K(m)}, \quad a=2 m \kappa^{2}
$$

and

$$
V=\Delta-6 d-2 a\left\{\frac{2-m}{m}-\frac{3 E(m)}{m K(m)}\right\} .
$$

Here $\operatorname{cn}(x ; m)$ is the Jacobi elliptic function of modulus $m$ and $K(m), E(m)$ are the elliptic integrals of the first and second kind, respectively (where $0<m<1$ ), $a$ is the wave amplitude, $d$ is the mean level, and $V$ is the wave speed. The spatial period is $2 K(m) / \kappa$. This family of solutions contains three free parameters, which are chosen from the set $\{a, \kappa, V, d, m\}$. As $m \rightarrow 1, \operatorname{cn}(x ; m) \rightarrow \operatorname{sech}(x)$ and then the cnoidal wave (B.3) becomes a solitary wave, riding on a background level $d$. On the other hand, as $m \rightarrow 0, \operatorname{cn}(x ; m) \rightarrow \cos x$ and so the cnoidal wave (B.3) collapses to a linear sinusoidal wave (note that in this limit $a \rightarrow 0$ ).

The asymptotic method of $[22,32]$ is to let expression (B.3) describe a modulated periodic wave train in which the amplitude $a$, the mean level $d$, the speed $V$ and the wave number $\kappa$ are all slowly varying functions of $x$ and $t$. The outcome is a set of three nonlinear hyperbolic equations for three of the available free parameters, chosen from the set $\{a, \kappa, V, d, m\}$ or, rather better, from an appropriate combination of them. These equations are often called the Whitham equations. The relevant asymptotic 
solution corresponding to the initial condition (B.2) is then constructed in terms of the similarity variable $x / t$, and is given by

$$
\begin{gathered}
\Delta-\frac{x}{t}=2 A_{0}\left\{1+m-\frac{2 m(1-m) K(m)}{E(m)-(1-m) K(m)}\right\} \\
\text { for }-6 A_{0}<\Delta-\frac{x}{t}<4 A_{0}, \\
a=2 A_{0} m, \quad d=A_{0}\left\{m-1+\frac{2 E(m)}{K(m)}\right\} .
\end{gathered}
$$

A plot of this expression is shown in Figure 10. Ahead of the wave train where $x / t<\Delta-4 A_{0}, A=0$, and at this end, $m \rightarrow 1, a \rightarrow 2 A_{0}$ and $d \rightarrow 0$; the leading wave is a solitary wave of amplitude $2 A_{0}$ relative to a mean level of 0 . Behind the wave train where $x / t>\Delta+6 A_{0}, A=A_{0}$, and at this end $m \rightarrow 0, a \rightarrow 0$ and $d \rightarrow A_{0}$; the wave train is now sinusoidal with a wave number $\kappa$ given by $\kappa^{2}=A_{0}$ (this holds throughout the wave train, so all waves have the same spatial wavelength). Further, it can be shown that on any individual crest in the wave train, $m \rightarrow 1$ as $t \rightarrow \infty$. In this sense, the undular bore evolves into a train of solitary waves.

If $A_{0}<0$ in the initial condition (B.3), then an "undular bore" solution analogous to that described by (B.3), (B.5) does not exist. Instead, the asymptotic solution is a rarefraction wave,

$$
\begin{aligned}
A=0 & \text { for } x<\Delta t, \\
A=\frac{1}{6}\left(\Delta-\frac{x}{t}\right) & \text { for } 6 A_{0}<\Delta-\frac{x}{t}<0, \\
A=A_{0} & \text { for } \Delta-\frac{x}{t}<6 A_{0}<0 .
\end{aligned}
$$

Small oscillatory wave trains are needed to smooth out the discontinuities in $A_{x}$ at the corners (see [22]).

The corresponding solutions when the initial condition is instead

$$
A=-A_{0} H(-x)
$$

can be obtained from the above by the transformation

$$
\hat{A}=A+A_{0}, \quad \hat{x}=x-6 A_{0} t,
$$

which transforms the initial-value problem (B.8) for (B.1) into the initial-value problem (B.2) for (B.1).

\section{References}

[1] T. R. Akylas, "On the excitation of long nonlinear water waves by moving pressure distribution", J. Fluid Mech. 141 (1984) 455-466.

[2] B. J. Binder, F. Dias and J.-M. Vanden-Broeck, "Steady free-surface flow past an uneven channel bottom", Theor. Comput. Fluid Dyn. 20 (2006) 125-144. 
[3] B. J. Binder, J.-M. Vanden-Broeck and F. Dias, "Forced solitary waves and fronts past submerged obstacles", Chaos 15 (2005) 037106.

[4] S. R. Clarke and R. H. J. Grimshaw, "Resonantly generated internal waves in a contraction", J. Fluid Mech. 274 (1994) 139-161.

[5] S. L. Cole, "Transient waves produced by flow past a bump", Wave Motion 7 (1985) 579-587.

[6] F. Dias and J.-M. Vanden-Broeck, "Generalized critical free surface flows", J. Engrg. Math. 42 (2002) 291-301.

[7] F. Dias and J.-M. Vanden-Broeck, "Steady two-layer flows over an obstacle", Philos. Trans. R. Soc. Ser. A 360 (2002) 2137-2154.

[8] F. Dias and J.-M. Vanden-Broeck, "Trapped waves between submerged obstacles", J. Fluid Mech. 509 (2004) 93-102.

[9] F. Dias and J.-M. Vanden-Broeck, "Two-layer hydraulic falls over an obstacle", Eur. J. Mech. B Fluids 23 (2004) 879-898.

[10] B. K. Ee and S. R. Clarke, "Weakly dispersive hydraulic flows in a contraction: parametric solutions and linear stability analysis", Phys. Fluids 19 (2007) 056601.

[11] B. K. Ee and S. R. Clarke, "Weakly dispersive hydraulic flows in a contraction: nonlinear stability analysis", Wave Motion 45 (2008) 927-939.

[12] B. K. Ee, R. H. J. Grimshaw, D.-H. Zhang and K. W. Chow, "Steady transcritical flow over a hole: parametric map of solutions of the forced Korteweg-de Vries equation", Phys. Fluids 22 (2010) 056602.

[13] G. A. El, R. H. J. Grimshaw and N. F. Smyth, "Transcritical shallow-water flow past topography: finite-amplitude theory", J. Fluid Mech. 640 (2009) 187-214.

[14] R. C. Ertekin, W. C. Webster and J. V. Wehausen, "Ship generated solitons", Proc. 15th Symp. Naval Hydrodyn., Hamburg (National Academy Press, Washington, DC, 1984) 347-364.

[15] R. C. Ertekin, W. C Webster and J. V. Wehausen, "Waves caused by a moving disturbance in a shallow channel of finite width", J. Fluid Mech. 169 (1986) 275-292.

[16] R. H. J. Grimshaw, K. H. Chan and K. W. Chow, "Transcritical flow of a stratified fluid: the forced extended Korteweg-de Vries model”, Phys. Fluids 14 (2002) 755-774.

[17] R. Grimshaw, E. Pelinovsky, T. Talipova and A. Kurkina, "Internal solitary waves: propagation, deformation and disintegration", Nonlinear Proc. Geoph. 17 (2010) 633-649.

[18] R. H. J. Grimshaw and N. F. Smyth, "Resonant flow of a stratified fluid over topography", J. Fluid Mech. 169 (1986) 429-464.

[19] R. H. J. Grimshaw, D.-H. Zhang and K. W. Chow, "Generation of solitary waves by transcritical flow over a step", J. Fluid Mech. 587 (2007) 235-254.

[20] R. H. J. Grimshaw, D.-H. Zhang and K. W. Chow, "Transcritical flow over a hole", Stud. Appl. Math. 122 (2009) 235-248.

[21] J. Grue, H. A. Friis, E. Palm and P.-O. Rusas, "A method for computing unsteady fully nonlinear interfacial waves", J. Fluid Mech. 351 (1997) 223-252.

[22] A. V. Gurevich and L. P. Pitaevskii, "Nonstationary structure of a collisionless shock wave", Sov. Phys. JETP 38 (1974) 291-297.

[23] D. B. Huang, O. J. Sibul, W. C. Webster, J. V. Wehausen, D. M. Wu and T. Y Wu, "Ships moving in the transcritical range", Proc. Conf. on Behaviour of Ships in Restricted Waters, Varna, Bulgaria (1982) 26-1-26-10.

[24] S. J. Lee, G. T. Yates and T. Y. Wu, "Experiments and analyses of upstream-advancing solitary waves generated by moving disturbances", J. Fluid Mech. 199 (1989) 569-593.

[25] J. Lighthill, Waves in fluids (Cambridge University Press, Cambridge, 1978).

[26] T. R. Marchant and N. F. Smyth, "The extended Korteweg-de Vries equation and the resonant flow of a fluid over topography", J. Fluid Mech. 221 (1990) 263-288.

[27] C. C. Mei, "Radiation of solitons by slender bodies advancing in a shallow channel", J. Fluid Mech. 162 (1986) 53-67.

[28] W. K. Melville and K. R. Helfrich, "Transcritical two-layer flow over topography", J. Fluid Mech. 178 (1987) 31-52. 
[29] A. Porter and N. F. Smyth, "Modelling the morning glory of the Gulf of Carpentaria", J. Fluid Mech. 454 (2002) 1-20.

[30] N. F. Smyth, "Modulation theory solution for resonant flow over topography", Proc. R. Soc. A 409 (1987) 79-97.

[31] J. G. Thews and L. Landweber, "The influence of shallow water on the resistance of a cruiser model", US Experimental Model Basin, Washington, DC, 1934, Report 408.

[32] G. B. Whitham, Linear and nonlinear waves (Wiley, New York, 1974).

[33] T. Y. Wu, "Generation of upstream advancing solitons by moving disturbances", J. Fluid Mech. 184 (1987) 75-99.

[34] D. M. Wu and T. Y. Wu, "Three-dimensional nonlinear long waves due to moving surface pressure", Proc. 14th Symp. Naval Hydrodyn. (National Academy Press, Washington, DC, 1982) $103-129$.

[35] D.-H. Zhang and A. T. Chwang, "On solitary waves forced by underwater moving objects", J. Fluid Mech. 389 (1999) 119-135.

[36] D.-H. Zhang and A. T. Chwang, "Generation of solitary waves by forward- and backward-step bottom forcing", J. Fluid Mech. 432 (2001) 341-350. 